\title{
Crossover model for the work of critical cluster formation in nucleation theory
}

\author{
V. I. Kalikmanov ${ }^{\text {a) }}$ \\ University of Delft, Department of Geotechnology, Laboratory of Applied Geophysics \& Petrophysics, \\ P.O. Box 5028, 2600 GA, Delft, The Netherlands
}

(Received 6 July 2004; accepted 20 August 2004)

\begin{abstract}
We propose a relation for the work of critical cluster formation in nucleation theory $W$ for the systems with long-range interparticle interactions. The method of bridge functions is used to combine the system behavior at sufficiently small quenches, adequately predicted by the classical nucleation theory, with nonclassical effects at deep quenches in the vicinity of the thermodynamic spinodal, described within the framework of the field theoretical approach with an appropriate Ginzburg-Landau functional. The crossover between the two types of nucleation behavior takes place in the vicinity of the kinetic spinodal where the lifetime of a metastable state is of the order of the relaxation time to local equilibrium. We argue that the kinetic spinodal corresponds to the minimum of the excess number of molecules in the critical cluster. This conjecture leads to the form of $W$ containing no adjustable parameters. The barrier scaling function $\Gamma=W / W^{\mathrm{cl}}$, where $W^{\mathrm{cl}}$ is the classical nucleation barrier, depends parametrically on temperature through the dimensionless combination of material properties. The results for argon nucleation are presented. () 2004 American Institute of Physics. [DOI: 10.1063/1.1806404]
\end{abstract}

\section{INTRODUCTION}

Scaling relations play an important role in nucleation theory increasing our ability to describe nucleation phenomena in complex substances for which microscopic description becomes intractable in view of the lack of knowledge of intermolecular interactions. A number of such approaches appeared in recent years. ${ }^{1}$ The main quantity of interest in these models is a barrier scaling function - the ratio of the nucleation barrier $W$ to its value $W^{\mathrm{cl}}$ predicted by the classical nucleation theory (CNT)

$$
\Gamma=\frac{W}{W^{\mathrm{cl}}} .
$$

The most recent model proposed by Kashchiev ${ }^{2}$ predicts $\Gamma$ to be a universal function of the quench depth, independent of either the material properties or temperature. This attractive feature of the theory of Kashchiev ${ }^{2}$ is, however, overshadowed by a number of controversial statements used in its formulation as discussed recently in Ref. 3.

The CNT assumes that the system is in a metastable state (e.g., supersaturated vapor for the case of gas-liquid transition) and its thermodynamics can be described by quasiequilibrium theory. This assumption leads to the capillarity approximation which presents the free energy barrier for a cluster formation as a sum of bulk and surface terms. The latter is a cost to form a droplet surface characterized by a plain layer surface tension $\gamma_{\infty}$ which is independent of the quench depth (supersaturation). A cluster (droplet) is assumed to be a compact spherical object. It is established that the nucleation behavior at low supersaturations is well described by the CNT.

${ }^{a)}$ Electronic mail: V.Kalikmanov@tnw.tudelft.nl
At deep quenches the system becomes unstable; in the theory of phase transitions the boundary between metastable and unstable region is given by a thermodynamic spinodal being a locus of points corresponding to a divergent compressibility. Rigorously speaking the transition from metastable to unstable states does not reduce to a sharp line but rather represents a region of a certain width which depends on the range of interparticle interactions. ${ }^{4}$ Nucleation in this region cannot be described by the CNT and a more general formalism is needed. A field theoretical approach to nucleation pioneered by Cahn and Hilliard ${ }^{5}$ and developed by Langer ${ }^{6}$ is based on the Ginzburg-Landau theory of phase transitions. Langer showed that if interparticle interactions are short-range the nucleating droplet has still a compact form and can be characterized by a sharp interface in agreement with the CNT predictions.

Langer's model, however, cannot be applied to systems with long-range interactions. In this case one can describe the system behavior in the framework of a mean-field approach leading to an existence of a well-defined (mean-field) spinodal. Mean-field theory becomes asymptotically accurate in the limit of infinite-range interactions and hence a spinodal exists in the same limit. Therefore, for systems with a sufficiently long-range interaction potential the concept of a spinodal can be applicable. Note in this respect that a spinodal is unambiguously determined for van der Waals fluids. At the spinodal the surface tension vanishes which means that capillary forces can no longer sustain the compact form of a droplet. Indeed, Klein and co-workers ${ }^{7,8}$ showed that clusters in the vicinity of a spinodal are ramified fractal objects.

When fluctuations are taken into account the kinetic considerations show that the lifetime of a metastable state de- 
creases becoming of the order of the relaxation time of the system to local equilibrium before the thermodynamic spinodal is reached. This situation corresponds to a kinetic spinodal $^{9}$ which is a physical crossover between metastable and unstable states. Beyond the kinetic spinodal the concept of quasiequilibrium, lying in the heart of the CNT, becomes inapplicable.

In the present paper we discuss nucleation in the systems with long-range interaction potentials focusing on the gasliquid transition. We use the method of bridge functions together with plausible physical arguments to combine the CNT behavior at low supersaturations with nonclassical spinodal effects at high supersaturations. We argue that the kinetic spinodal corresponds to the minimum of the excess number of molecules in the critical cluster over that present in the mother phase. This conjecture leads to the nucleation barrier $W$ which is the simplest smooth interpolation between the two regimes. At the same time $W$ and $\Gamma$ parametrically depend on temperature through the material parameters, such as saturation pressure, plain interface surface tension, equilibrium liquid density, and the vapor pressure at the mean-field spinodal.

The paper is organized as follows. In Sec. II we briefly discuss the CNT result for the nucleation barrier using the Gibbs approach to thermodynamics of curved surfaces. In Sec. III A we summarize the field-theoretical description of nucleation near the mean-field thermodynamic spinodal using the Ginzburg-Landau free energy functional and determine the scaling laws for the size and amount of particles in the critical cluster in this domain. Section III B is devoted to the role of fluctuations in the nucleation phenomenon which lead to a concept of a kinetic spinodal. Using the method of bridge functions, the condition of kinetic spinodal and formulating an ansatz for the crossover between the CNTdominated regime and the spinodal-dominated regime, we construct in Sec. IV the nucleation barrier and the barrier scaling function for the entire range of supersaturations. In Sec. V we apply our theory to argon for which the thermodynamic spinodal is obtained from the van der Waals equation of state. Appendix presents calculations of the supersaturated vapor density and the thermodynamic spinodal for van der Waals fluids.

\section{GIBBS THERMODYNAMICS OF CURVED SURFACES AND CLASSICAL NUCLEATION THEORY}

In this section we briefly discuss the CNT prediction for the free energy barrier paying attention to the underlying assumptions. Consider a system "droplet+ vapor" put in contact with a reservoir at a temperature $T<T_{c}$ ( $T_{c}$ is the critical temperature) and pressure $p^{v}$ which exceeds the coexistence (saturation) pressure $p_{\text {sat }}(T)$. The system thus finds itself in a metastable state; the degree of metastability is conventionally described by the supersaturation ratio:

$$
S=\frac{p^{v}}{p_{\text {sat }}(T)} .
$$

If $S$ is not high the lifetime of the metastable state is much larger than the relaxation time of the system to its local equi- librium and one can use quasiequilibrium theory considering a droplet to be in equilibrium with the surrounding supersaturated vapor and applying the Gibbs equilibrium thermodynamics of curved surfaces ${ }^{10,11}$ to this metastable state. Assuming that an arbitrary chosen dividing surface is spherical with a radius $R$, one obtains the reversible work of a droplet formation in the capillarity approximation ${ }^{10}$

$$
W=-\Delta p \frac{4 \pi}{3} R^{3}+4 \pi R^{2} \gamma(R),
$$

where the surface tension $\gamma(R)$ refers to the chosen dividing surface; $\Delta p=p^{l}-p^{v}, p^{l}$ being the pressure of the bulk liquid held at the same temperature $T$ and the same chemical potential $\mu$ as the supersaturated vapor: $\mu=\mu^{v}\left(p^{v}\right)=\mu^{l}\left(p^{l}\right)$. For the surface of tension with $R=R_{t}, \gamma\left(R_{t}\right)=\gamma_{t}$ taking into account the Laplace equation $\Delta p=2 \gamma_{t} / R_{t}$, we find from (2): $W=(16 \pi / 3) \gamma_{t}^{3} / \Delta p^{2}$. Since $\gamma_{t}$ is not known, the CNT approximates it by the plain interface value $\gamma_{\infty}(T)$, resulting in the classical nucleation barrier

$$
W^{\mathrm{cl}}=\frac{16 \pi}{3} \frac{\gamma_{\infty}^{3}}{(\Delta p)^{2}} .
$$

The nucleation barrier is the leading order contribution to the nucleation rate $J$ which is the number of critical clusters formed per unit volume per unit time:

$$
J=K e^{-W / k_{\mathrm{B}} T} .
$$

Here $k_{\mathrm{B}}$ is the Boltzmann constant and $K$ is the kinetic prefactor. In the CNT kinetics of cluster formation is described by a set of elementary processes which change the size of a cluster by attachment to its surface or loss from its surface of one molecule. The kinetic prefactor can be well approximated by ${ }^{12}$

$$
K \approx \frac{\left(\rho^{v}\right)^{2}}{\rho^{l}} \sqrt{\frac{2 \gamma_{\infty}}{\pi m_{1}}}
$$

where $m_{1}$ is the mass of a molecule. Assuming that the liquid is incompressible and the vapor is ideal (which is a reasonable assumption not too close to $T_{c}$ ) we write down the Kelvin equation ${ }^{13}$ relating $\Delta p$ to $S$,

$$
\Delta p=\rho_{\mathrm{eq}}^{l} k_{\mathrm{B}} T \ln S,
$$

where $\rho_{\text {eq }}^{l}(T)$ is the equilibrium liquid number density. At the spinodal the supersaturation reaches its maximum $S_{\mathrm{sp}}$ corresponding to the maximum of $\Delta p$ :

$$
\Delta p_{\mathrm{sp}}=\rho_{\mathrm{eq}}^{l} k_{\mathrm{B}} T \ln S_{\mathrm{sp}} .
$$

Introducing another useful measure of metastability

$$
\eta=\Delta p / \Delta p_{\mathrm{sp}}=\ln S / \ln S_{\mathrm{sp}}, \quad 0 \leqslant \eta \leqslant 1
$$

we present (3) as

$$
W^{\mathrm{cl}}=c_{b} \frac{1}{\eta^{2}}, \quad c_{b}(T)=\frac{16 \pi}{3} \frac{\gamma_{\infty}^{3}}{\left(\Delta p_{\mathrm{sp}}\right)^{2}} .
$$

A general result in nucleation theory which holds for all cluster sizes down to atomic scale (where the capillarity approximation fails) and which is independent of a particular model is known as the nucleation theorem. ${ }^{14,15}$ It states that 
the excess number of molecules in the critical cluster over that present in the mother phase $\Delta n_{*}$ is related to the nucleation barrier via,

$$
\frac{\partial W}{\partial \Delta \mu}=-\Delta n_{*},
$$

where $\Delta \mu=\mu^{v}\left(p^{v}\right)-\mu^{l}\left(p^{v}\right)$. Using the standard thermodynamic relationships this expression can be rewritten as

$$
\left(\frac{\rho_{\mathrm{eq}}^{l}}{\Delta p_{\mathrm{sp}}}\right) \frac{\partial W}{\partial \eta}=-\Delta n_{*} .
$$

Applying the nucleation theorem to the CNT we have,

$$
\Delta n_{*}^{\mathrm{cl}}=\left(\frac{2 c_{b} \rho_{\mathrm{eq}}^{l}}{\Delta p_{\mathrm{sp}}}\right) \eta^{-3}
$$

which in combination with the Laplace equation yields the scaling relation for the critical cluster,

$$
\Delta n_{*}^{\mathrm{cl}} \sim R^{3}
$$

showing (in agreement with the assumptions made) that the critical cluster in the CNT is a compact spherical object.

It is tempting to apply the CNT to the entire domain 0 $\leqslant \eta \leqslant 1$. By doing so one would find that as spinodal is approached,

$$
\lim _{\eta \rightarrow 1} W^{\mathrm{cl}}=c_{b}=\text { const, } \quad \lim _{\eta \rightarrow 1} \Delta n_{*}^{\mathrm{cl}}=\frac{2 c_{b} \rho_{\mathrm{eq}}^{l}}{\Delta p_{\mathrm{sp}}}=\text { const. }
$$

On the other hand the field theoretical arguments, presented in the following section, supported by the density functional calculations, ${ }^{17}$ show that as the system approaches the meanfield spinodal the nucleation barrier vanishes, while $\Delta n_{*}$ diverges. Moreover, the nucleating cluster at the same limit is not a compact object but a fractal, correspondingly a dividing surface cannot be characterized by a single parameter $(R)$. These results, however, are not in contradiction with the classical theory since the latter is simply not designed to be applicable in the spinodal region where its basic assumption-the concept of quasiequilibrium-becomes meaningless. Note in this respect that the statement used by some authors (see Ref. 2), that the CNT is thermodynamically inconsistent because it predicts a finite value of $W$ at the spinodal, is irrelevant.

\section{NONCLASSICAL NUCLEATION}

\section{A. Nucleation in the vicinity of the thermodynamic spinodal}

To analyze the nucleation behavior in the vicinity of the thermodynamic spinodal we apply field theoretical considerations following Refs. 5, 6 and 8. We start with Landau expansion $^{18}$ for the free energy density in powers of the order parameter $m$.

$$
g=g_{0}+\frac{a}{2} m^{2}+\frac{b}{4} m^{4}-m h,
$$

where

$$
a=a_{0} t, \quad t \equiv\left(T-T_{c}\right) / T_{c}, \quad a_{0}>0, b>0,
$$

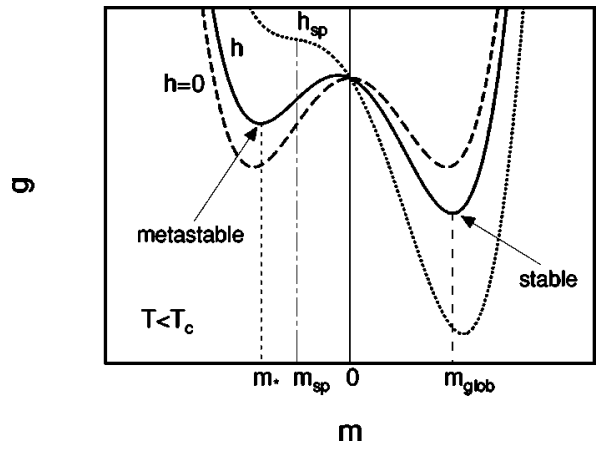

FIG. 1. Schematic plot of Landau free energy density $g$ for $T<T_{c}$. At $h$ $=0$ (long dashed line) there are two equal minima corresponding to the coexisting states. At $0<h<h_{\text {sp }}$ (solid line) the left, local, minimum $m_{*}$ corresponds to a metastable state (supersaturated vapor), while the right, global, minimum $m_{\text {glob }}$ refers to a stable state (liquid); the two states are separated by the energy barrier. At $h=h_{\text {sp }}$ (short dashed line) the local minimum disappears-this is the case of spinodal decomposition.

$h$ is the external field conjugate to $m$. For the gas-liquid transition $m=\rho-\rho_{c}$ (where $\rho_{c}$ is the critical density) and $h$ $=\Delta \mu$; at the spinodal $h=h_{\mathrm{sp}}$, while at the binodal $h=0$. Below $T_{c}, a<0$ and the free energy density has a doublewell structure with the local minimum at $m=m_{*}$ corresponding to the given metastable state and the global minimum at $m=m_{\text {glob }}$ corresponding to the thermodynamically stable liquid state to which the system evolves (see Fig. 1). The two minima are separated by the energy barrier. Standard analysis ${ }^{19,20}$ yields

$$
m_{*}(h)=2 \sqrt{\frac{s}{3 b}} \cos \left(\alpha+\frac{2 \pi}{3}\right),
$$

where $s=-a>0$ and

$$
\cos 3 \alpha=\frac{3 \sqrt{3}}{2} \frac{b^{1 / 2}}{s^{3 / 2}} h, \quad 0 \leqslant \alpha \leqslant \frac{\pi}{6} .
$$

The maximum supersaturation corresponds to $h_{\mathrm{sp}}^{2}$ $=(4 / 27) s^{3} / b$; at this field the local minimum $m_{\text {sp }}$ becomes an inflection point - this is the case of spinodal decomposition. Thus, nucleation takes place for the field interval 0 $<h^{2}<h_{\mathrm{sp}}^{2}$. For the states close to $m_{*}$ the free energy density can be expanded in powers of $\phi=\left(m-m_{*}\right) / m_{*}$ :

$$
g=g_{*}+\frac{b_{2}}{2} \phi^{2}-\frac{b_{3}}{3} \phi^{3}+O(\phi)^{4}
$$

where $g_{*}$ is the free energy density of the metastable state $m_{*}$ and

$$
b_{2}(h)=\left.m_{*}^{2} \frac{\partial^{2} g}{\partial m^{2}}\right|_{m=m *}, \quad b_{3}(h)=-\left.m_{*}^{3} \frac{1}{2} \frac{\partial^{3} g}{\partial m^{3}}\right|_{m=m *} .
$$

The term linear in $\phi$ vanishes since $m=m_{*}$ is a local minimum of $g$. At the spinodal

$$
b_{2}\left(h=h_{\mathrm{sp}}\right)=0, \quad b_{3}\left(h=h_{\mathrm{sp}}\right)>0 .
$$

Let us study the system behavior near the mean-field spinodal (i.e. when $h$ is close to $h_{\mathrm{sp}}$ ) using an appropriate Ginzburg-Landau free energy functional which describes the 
state of the system undergoing a first-order phase transition characterized by a conserved scalar order parameter $\phi(\mathbf{r})$ $=\left[m(\mathbf{r})-m_{*}\right] / m_{*} \cdot{ }^{21}$ Using Eq. (11) this functional reads,

$$
\mathcal{F}[\phi(r)]=\mathcal{F}_{*}+\int d \mathbf{r}\left[\frac{c_{0}}{2}|\nabla \phi|^{2}+\frac{b_{2}}{2} \phi^{2}-\frac{b_{3}}{3} \phi^{3}\right],
$$

where $\mathcal{F}_{*}$ is the free energy of the metastable state $m$ $=m_{*}$, out of which nucleation starts. The square-gradient term is an energy cost to create an interface between the phases; $c_{0}>0$ is related to the correlation length in the system. $^{22}$ Following Ref. 8 we associate the critical cluster with the saddle point of the free energy functional (13). If the saddle point $\phi(\mathbf{r})$ is found, its substitution into $\mathcal{F}$ yields the nucleation barrier

$$
W=\mathcal{F}-\mathcal{F}_{*}=\int d \mathbf{r}\left[\frac{c_{0}}{2}|\nabla \phi|^{2}+\frac{b_{2}}{2} \phi^{2}-\frac{b_{3}}{3} \phi^{3}\right] .
$$

To analyze this expression we proceed by performing a set of scaling transformation of the variables. Rescaling the order parameter $\phi_{1}=\left(b_{3} / c_{0}\right)^{1 / 3} \phi$ and denoting

$$
\epsilon=b_{2}\left(b_{3}^{2} c_{0}\right)^{-1 / 3}
$$

we rewrite (14) as

$$
W=c_{0} \int d \mathbf{r}\left[\frac{1}{2}\left(\frac{c_{0}}{b_{3}}\right)^{2 / 3}\left|\nabla \phi_{1}\right|^{2}+\frac{\epsilon}{2} \phi_{1}^{2}-\frac{1}{3} \phi_{1}^{3}\right] .
$$

The next transformation rescales the spatial coordinates $\mathbf{r}_{1}$ $=\left(b_{3} / c_{0}\right)^{-1 / 3} \mathbf{r}$ yielding

$$
W=b_{3} \int d \mathbf{r}_{1}\left[\frac{1}{2}\left|\nabla_{1} \phi_{1}\right|^{2}+\frac{\epsilon}{2} \phi_{1}^{2}-\frac{1}{3} \phi_{1}^{3}\right],
$$

where $\nabla_{1}=\partial / \partial \mathbf{r}_{1}$. And finally, further rescaling is useful,

$$
\phi_{1}=\epsilon \widetilde{\phi}, \quad \mathbf{r}_{1}=\epsilon^{-1 / 2} \widetilde{\mathbf{r}},
$$

with the help of which $W$ takes the form,

$$
W=\epsilon^{3 / 2} b_{3} \int d \widetilde{\mathbf{r}}\left[\frac{1}{2}|\widetilde{\nabla} \widetilde{\phi}|^{2}+\frac{1}{2} \widetilde{\phi}^{2}-\frac{1}{3} \widetilde{\phi}^{3}\right],
$$

where $\tilde{\nabla}=(\partial / \partial \widetilde{\mathbf{r}})$. The saddle point of the functional is given by the Euler-Lagrange equation:

$$
\widetilde{\nabla}^{2} \widetilde{\phi}=\widetilde{\phi}-\widetilde{\phi}^{2}
$$

The critical cluster is the nontrivial solution of Eq. (18) vanishing at infinity. The existence of such solutions was proved for sufficiently large bounded domains. ${ }^{23}$ Without presenting the full form of the solution it is instructive to study its behavior at large r, i.e., far from the center of mass of the cluster. In this domain the amplitude of the droplet is small and we can neglect the second term in Eq. (18) which leads to the equation

$$
\widetilde{\nabla}^{2} \widetilde{\phi}=\widetilde{\phi}, \quad \text { large } \tilde{r},
$$

whose spherically symmetric solution vanishing at infinity is the screened Coulomb function:

$$
\widetilde{\phi}=C_{>} \frac{e^{-\tilde{r}}}{\tilde{r}}, \text { large } \tilde{r},
$$

where $C_{>}$is a constant. Returning to the units of Eq. (16) it reads

$$
\phi_{1}\left(r_{1}\right)=C_{>} \sqrt{\epsilon} \frac{e^{-\sqrt{\epsilon} r_{1}}}{r_{1}}, \quad \text { large } r_{1} .
$$

Since $\phi_{1}\left(r_{1}\right)$ is the density fluctuation associated with a nucleus, its decay length

$$
R_{*} \approx \epsilon^{-1 / 2}, \quad \epsilon \rightarrow 0
$$

characterizes the size of the critical nucleus. The latter diverges as the spinodal is approached. In the same limit the nucleation barrier (17) decreases as

$$
W \sim \epsilon^{3 / 2}, \quad \epsilon \rightarrow 0 .
$$

Finally we must relate $\epsilon$ to the physical parameters of the system, i.e. to determine the scaling of $\epsilon$ near spinodal to the leading order in $\left(h-h_{\mathrm{sp}}\right)$; obviously $\epsilon\left(h=h_{\mathrm{sp}}\right)=0$. From Eq. (15) and the definition of $b_{2}$ it follows that $\epsilon$ is proportional to the curvature of the Landau free energy at the metastable state,

$$
\left.\epsilon \sim b_{2} \sim g_{2}(h) \equiv \frac{\partial^{2} g}{\partial m^{2}}\right|_{m=m *(h)}=-s+3 b m_{*}^{2} .
$$

Substituting $h=h_{\mathrm{sp}}-u$, where $u$ is a (small) deviation of the external field from its value at the spinodal, into Eqs. (9) and (10), we obtain to the leading order in $u$,

$$
g_{2}=2(3 b s)^{1 / 4} \sqrt{h_{\mathrm{sp}}-h} .
$$

Since $h_{\mathrm{sp}}-h \sim \Delta p_{\mathrm{sp}}-\Delta p=\Delta p_{\mathrm{sp}}(1-\eta)$, we have

$$
\epsilon \sim(1-\eta)^{1 / 2} \text {. }
$$

Substituting Eq. (21) into Eqs. (20) and (19) we find that in the vicinity of the thermodynamic spinodal the nucleation barrier vanishes as

$$
W_{\mathrm{sp}}=c_{\mathrm{sp}}(T)(1-\eta)^{3 / 4}, \quad c_{\mathrm{sp}}(T)>0, \quad \eta \rightarrow 1^{-},
$$

while the radial extent of the critical cluster diverges as

$$
R_{*}=R_{0}(1-\eta)^{-1 / 4}, \quad R_{0}(T)>0, \quad \eta \rightarrow 1^{-} .
$$

The excess number of molecules in the critical cluster in the spinodal region is found from the nucleation theorem (7):

$$
\Delta n_{*}=\frac{3}{4}\left(\frac{c_{\mathrm{sp}} \rho_{\mathrm{eq}}^{l}}{\Delta p_{\mathrm{sp}}}\right)(1-\eta)^{-1 / 4}, \quad \eta \rightarrow 1^{-} .
$$

Thus, near the spinodal $\Delta n_{*}$ diverges. Comparison of Eqs. (23) and (24) predicts the scaling,

$$
\Delta n_{*} \sim R_{*} \text {. }
$$

This result supports the conjecture of Klein ${ }^{7}$ that the critical cluster near the spinodal is a ramified fractal (chain-like) object. This is distinctly different from the scaling $\Delta n_{*}$ $\sim R^{3}$ near the binodal predicted by the CNT [cf. Eq. (8)].

Note, that the nucleation barrier proposed in Ref. 2,

$$
W_{K}=c_{b}\left(\frac{1}{\eta}+\frac{1}{2}\right)^{2}(1-\eta), \quad 0 \leqslant \eta \leqslant 1
$$

scales near the thermodynamic spinodal as

$$
W_{K}=\frac{9}{4} c_{b}(1-\eta), \quad \eta \rightarrow 1,
$$


which implies using the nucleation theorem that $\Delta n_{*, K}$ at the spinodal is finite

$$
\Delta n_{*, K}=\frac{9}{4}\left(\frac{c_{b} \rho_{\mathrm{eq}}^{l}}{\Delta p_{\mathrm{sp}}}\right)+O(1-\eta), \quad \eta \rightarrow 1^{-}
$$

in contradiction with the nonclassical results given by Eqs. (22) and (24).

\section{B. Role of fluctuations: Kinetic spinodal}

The condition $b_{2}=0$ describes the mean-field thermodynamic spinodal in the absence of fluctuations. However, their role in nucleation phenomena is important. One is facing the question: how deep the quench can be so that the concept of quasiequilibrium invoked in CNT can be considered still valid? To find an answer it is necessary to compare two characteristic times: (i) the time $t_{M}$ necessary to form a critical cluster which is a lifetime of the metastable state and (ii) the relaxation time $t_{R}$ during which the system settles in this state. The first quantity can be related to the nucleation rate by using its definition: $t_{M}=1 /(J V)$. To find $t_{R}$ one must study the dynamics of the metastable state. Since the order parameter $\phi(\mathbf{r})$ in the Ginzburg-Landau functional (13) is a conserved variable, its evolution is governed by the CahnHilliard dissipative dynamics: ${ }^{24}$

$$
\frac{\partial \phi}{\partial t}=\Gamma_{0} \nabla^{2} \frac{\delta \mathcal{F}}{\delta \phi}+\zeta
$$

where $\Gamma_{0}$ is a transport coefficient and $\zeta(\mathbf{r}, t)$ is a noise source (which models thermal fluctuations) satisfying

$$
\left\langle\zeta(\mathbf{r}, t) \zeta\left(\mathbf{r}^{\prime}, t^{\prime}\right)\right\rangle=-2 T \Gamma_{0} \nabla^{2} \delta\left(\mathbf{r}-\mathbf{r}^{\prime}\right) \delta\left(t-r^{\prime}\right)
$$

to ensure that the equilibrium distribution associated with Eq. (25) is given by the Boltzmann statistics. From the solution of Eqs. (25) and (13), obtained by Patashinskii and Shumilo $^{9}$ (see also Ref. 25), it follows that

$$
t_{R}=\frac{16 c_{0}}{\Gamma_{0} b_{2}^{2}},
$$

implying that when the system approaches the thermodynamic spinodal $\left(b_{2} \rightarrow 0\right)$ its relaxation time diverges. The relation between $t_{M}$ and $t_{R}$ established in Ref. 9 is

$$
t_{M}=t_{R}\left(\frac{4 \pi \chi}{\lambda_{0}}\right) \exp \left(\frac{\chi W}{k_{\mathrm{B}} T}\right),
$$

where

$$
\chi=\frac{\left(b_{2} c_{0}\right)^{3 / 2}}{k_{\mathrm{B}} T b_{3}^{2}},
$$

and $\lambda_{0} \approx 8.25$. Clearly, the concept of quasi-equilibrium is meaningful for the metastable states satisfying the requirement: $t_{M} \gg t_{R}$. In the opposite case this concept becomes irrelevant. The boundary between these two domains is called a kinetic spinodal ${ }^{9}$ and is defined by the condition $t_{M} \cong t_{R}$ yielding

$$
\frac{\chi W}{k_{\mathrm{B}} T}=1 .
$$

Using Eq. (26) it can be written as

$$
W=\frac{\left(k_{\mathrm{B}} T\right)^{2} b_{3}^{2}}{c_{0}^{3 / 2} b_{2}^{3 / 2}} .
$$

The quantities $b_{2}$ and $b_{3}$ are expressed in terms of the derivatives of the free energy as given by Eq. (12) and hence can be calculated given the equation of state:

$$
\begin{aligned}
& b_{2}=\left.\rho^{2} \frac{\partial \mu}{\partial \rho}\right|_{\rho=\rho^{v}}=\left.\rho \frac{\partial p}{\partial \rho}\right|_{\rho=\rho^{v}}, \\
& b_{3}=-\left.\frac{1}{2} \rho^{3} \frac{\partial^{2} \mu}{\partial \rho^{2}}\right|_{\rho=\rho^{v}}=b_{2}-\left.\frac{1}{2} \rho \frac{\partial b_{2}}{\partial \rho}\right|_{\rho=\rho^{v}} .
\end{aligned}
$$

The parameter $c_{0}$ can be well approximated by ${ }^{25}$

$$
c_{0} \cong k_{\mathrm{B}} T \rho_{c}^{1 / 3} .
$$

Hence, all parameters in Eq. (27) are temperature dependent material properties which can be determined from the equation of state $p=p(\rho, T)$. In particular from Eq. (28) it follows that $b_{2}$ is the inverse isothermal compressibility of the vapor at the metastable state.

\section{NUCLEATION BARRIER: A CROSSOVER MODEL}

At low supersaturations, where the capillarity approximation holds, the CNT expression (6) accurately describes the nucleation barrier; at high supersaturations in the spinodal region the capillarity approximation fails and $W$ is given by the field theoretical model (22). To obtain the nucleation barrier for the entire range of $0 \leqslant \eta \leqslant 1$ we propose a smooth interpolation between the two regions introducing a bridge function $B(\eta)$ :

$$
W(\eta)=c_{b} \eta^{-2}+c_{\mathrm{sp}}(1-\eta)^{3 / 4}+B(\eta),
$$

which should be smooth and satisfy the boundary conditions,

$$
\begin{aligned}
& \eta^{2} B(\eta) \rightarrow 0 \quad \text { for } \eta \rightarrow 0^{+}, \\
& (1-\eta)^{-3 / 4}\left[c_{b}+B(\eta)\right] \rightarrow 0 \text { for } \eta \rightarrow 1^{-} .
\end{aligned}
$$

Obviously, these constraints do not define $B(\eta)$ in a unique way. We choose the simplest form $B(\eta)=-c_{b}$ satisfying both boundary conditions. Then from Eq. (31),

$$
W=c_{b}\left[\eta^{-2}+\kappa(1-\eta)^{3 / 4}-1\right], \quad 0 \leqslant \eta \leqslant 1,
$$

with the yet unknown dimensionless parameter $\kappa$ $\equiv c_{\text {sp }}(T) / c_{b}(T)>0$. Let us consider the excess number of molecules in the critical cluster applying the nucleation theorem (7) to (32),

$$
\Delta n_{*}=\left(\frac{c_{b} \rho_{\mathrm{eq}}^{l}}{\Delta p_{\mathrm{sp}}}\right)\left[2 \eta^{-3}+\frac{3}{4} \kappa(1-\eta)^{-1 / 4}\right], \quad 0 \leqslant \eta \leqslant 1 .
$$

The qualitative behavior of $\Delta n_{*}(\eta)$ is shown in Fig. 2. We distinguish two domains of the metastable states: the "CNT domain" and the "spinodal domain." In the CNT domain $\Delta n_{*}$ is decreasing with the quench depth $\eta$ while in the spinodal domain $\Delta n_{*}(\eta)$ is increasing tending to infinity as the thermodynamic spinodal is approached. At some $\bar{\eta}$ it reaches the minimum $\overline{\Delta n_{*}}$. It is plausible to assume that the CNT-dominated regime covers the range of quench depths up to $\bar{\eta}$ and at $\bar{\eta}$ the spinodal-dominated behavior takes over. 


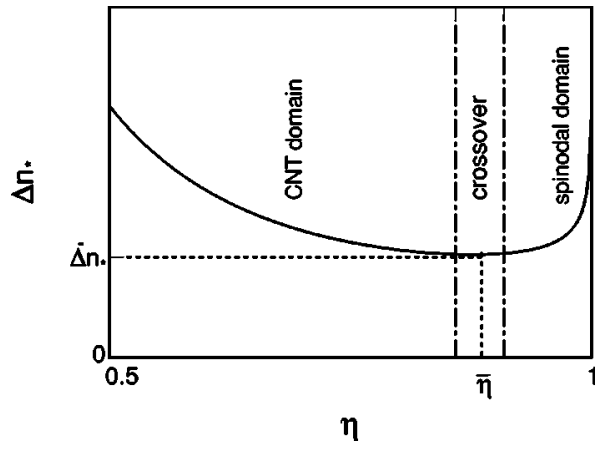

FIG. 2. Excess number of molecules in the critical cluster $\Delta n_{*}$ as a function of the quench depth $\eta$ given by Eq. (33). In the CNT domain $\Delta n_{*}$ is decreasing while in the spinodal domain it is increasing tending to infinity; at $\bar{\eta}$ it reaches the minimum value $\overline{\Delta n_{*}}$.

We associate the crossover between these two domains with the kinetic spinodal. This ansatz stems from the argument that the kinetic spinodal is the physical limit of applicability of quasiequilibrium approach on which the CNT is based. These considerations lead to the set of equations:

$$
\begin{aligned}
& \left.\frac{d \Delta n_{*}}{d \eta}\right|_{\bar{\eta}}=0, \\
& W(\bar{\eta})=\frac{\left(k_{\mathrm{B}} T\right)^{2}\left[b_{3}(\bar{\eta})\right]^{2}}{c_{0}^{3 / 2}\left[b_{2}(\bar{\eta})\right]^{3 / 2}} .
\end{aligned}
$$

Equation (34) yields

$$
\kappa=\frac{32(1-\bar{\eta})^{5 / 4}}{\bar{\eta}^{4}} .
$$

Substituting it into Eq. (35) we obtain the crossover equation determining $\bar{\eta}$ :

$$
\frac{1}{\bar{\eta}^{2}}+\frac{32(1-\bar{\eta})^{2}}{\bar{\eta}^{4}}-1=\frac{\left(k_{\mathrm{B}} T\right)^{2}\left[b_{3}(\bar{\eta})\right]^{2}}{c_{b} c_{0}^{3 / 2}\left[b_{2}(\bar{\eta})\right]^{3 / 2}} .
$$

The crossover is expected to be close to the thermodynamic spinodal [later we verify this assumption analyzing the solution of Eq. (37)]. It is then plausible to expand $b_{2}$ and $b_{3}$ in density in the vicinity of the spinodal keeping the linear order terms. For $b_{2}$ we have

$$
b_{2}=\xi\left(\rho^{v}-\rho_{\mathrm{sp}}^{v}\right), \quad \xi=\left.\frac{\partial b_{2}}{\partial \rho}\right|_{\mathrm{sp}}
$$

[the constant term vanishes since $b_{2}\left(\rho_{\mathrm{sp}}^{v}\right)=0$ ]. From Eqs. (29) and (38)

$$
b_{3}=\xi\left(\frac{1}{2} \rho^{v}-\rho_{\mathrm{sp}}^{v}\right) .
$$

Both $b_{2}$ and $b_{3}$ have the dimensionality of pressure, therefore it is convenient to rewrite them using the reduced variables $\rho^{*}=\rho^{v} / \rho_{c}, T^{*}=T / T_{c}, p^{*}=p^{v} / p_{c}$ (the subscript $c$ refers to the critical point):

$$
b_{2}=p_{c} \Phi_{2}\left(\rho^{*}, T^{*}\right), \quad b_{3}=p_{c} \Phi_{3}\left(\rho^{*}, T^{*}\right),
$$

where

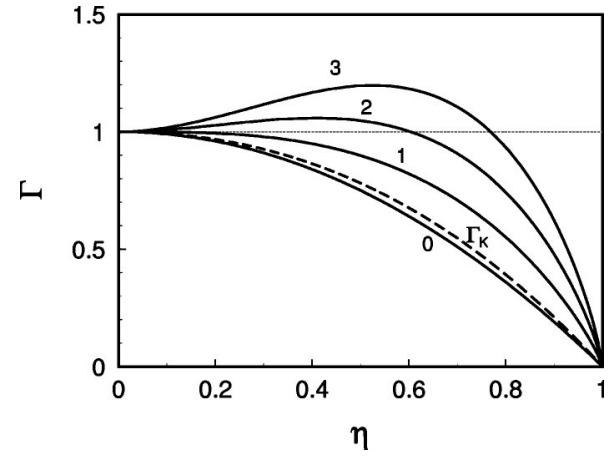

FIG. 3. Barrier scaling function (46) (solid lines); labels are the corresponding values of $\kappa$. Also shown is Kashchiev's function $\Gamma_{K}^{2}$ given by Eq. (47) (dashed line).

$\Phi_{2}\left(\rho^{*}, T^{*}\right)=\omega\left(\rho_{\mathrm{sp}}^{*}-\rho^{*}\right), \quad \Phi_{3}\left(\rho^{*}, T^{*}\right)=\frac{\omega}{2}\left(2 \rho_{\mathrm{sp}}^{*}-\rho^{*}\right)$,

and

$$
\omega\left(T^{*}\right)=-\left(\frac{\rho_{c}}{p_{c}}\right) \xi=-\left(\frac{\rho_{c}}{p_{c}}\right)\left(\rho \frac{\partial^{2} p}{\partial \rho^{2}}\right)_{\rho=\rho_{\mathrm{sp}}^{v}}>0 .
$$

Substitution of Eqs. (40) and (41) into Eq. (37) yields the crossover equation in the form,

$$
\frac{1}{\bar{\eta}^{2}}+\frac{32(1-\bar{\eta})^{2}}{\bar{\eta}^{4}}-1=\frac{\sqrt{\omega Z_{c}}}{4 \Lambda} \frac{\left[\left(2 \rho_{\mathrm{sp}}^{*}-\rho^{*}(\bar{\eta})\right]^{2}\right.}{\left[\rho_{\mathrm{sp}}^{*}-\rho^{*}(\bar{\eta})\right]^{3 / 2}},
$$

where $Z_{c}=p_{c} /\left(\rho_{c} k_{B} T_{c}\right)$ is the critical compressibility factor, and

$$
\Lambda(T)=\frac{16 \pi}{3} \frac{\gamma_{\infty}^{3}}{k_{\mathrm{B}} T \Delta p_{\mathrm{sp}}^{2}} .
$$

Having solved it for $\bar{\eta}$ one can estimate the excess number of molecules in the critical cluster at the crossover. From Eqs. (33) and (36) we find

$$
\overline{\Delta n_{*}}=\Delta n_{*}(\bar{\eta})=\left(\frac{2 \Lambda}{\ln S_{\mathrm{sp}}}\right)\left(\frac{12-11 \bar{\eta}}{\bar{\eta}^{4}}\right) .
$$

Finally, the barrier scaling function (1) reads

$$
\Gamma(\eta)=1-\eta^{2}+\kappa \eta^{2}(1-\eta)^{3 / 4},
$$

where $\kappa$ is given by Eq. (36) with $\bar{\eta}$ being a solution of the crossover equation (43). We stress that $\Gamma$ parametrically depends on temperature through $\kappa$.

The behavior of $\Gamma(\eta)$ for various values of $\kappa$ is shown in Fig. 3. If $\kappa<1: \Gamma<1$ for all values of supersaturation implying that the nucleation rate $J$ exceeds the rate $J^{\text {cl }}$ predicted by the CNT. In the opposite case of $\kappa>1$, the function $\Gamma$ exceeds unity at some range of supersaturations corresponding to $0 \leqslant \eta \leqslant \eta_{*}$, beyond which it becomes less than unity. In Fig. 3 we also show Kashchiev's universal function ${ }^{2}$

$$
\Gamma_{K}(\eta)=\left(1+\frac{1}{2} \eta\right)^{2}(1-\eta),
$$




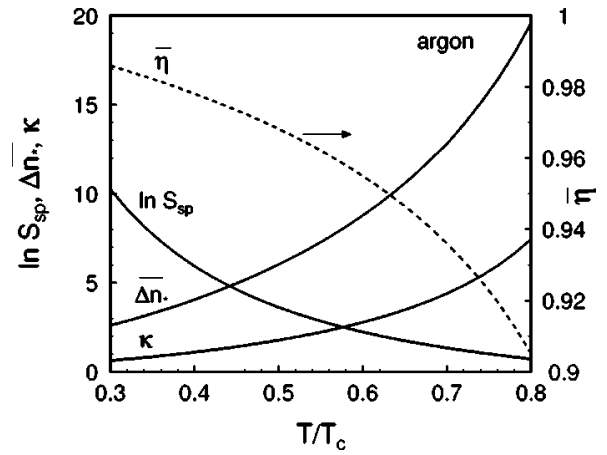

FIG. 4. Model parameters for argon. Solid lines (left $y$ axis): $S_{\mathrm{sp}}, \overline{\Delta n_{*}}, \kappa$. Dashed line (right $y$-axis) is the locus of crossover points $\bar{\eta}$.

which is close (though not identical) to our result when $\kappa$ $\approx 0.2$. Note that according to Eq. (47) the nucleation rate predicted by the model $^{2}$ exceeds the classical one for all temperatures and all supersaturations.

\section{RESULTS AND DISCUSSION}

We apply the present theory to argon. Empirical correlations for its bulk properties are given in Ref. 27 For the plain interface surface tension we use the general correlation for nonpolar substances. ${ }^{27}$ To a good approximation argon can be considered a van der Waals fluid. From the van der Waals equation of state we derive the vapor density at the supersaturated state $\rho^{v}\left(p^{v}, T\right)$ and the vapor part of the thermodynamic spinodal (see the Appendix). Figure 4 shows the model parameters $S_{\mathrm{sp}}, \overline{\Delta n_{*}}, \kappa$ for the temperature range $0.3<T / T_{c}<0.8$. The solution $\bar{\eta}$ of the crossover equation, shown on the right $y$ axis, is close to unity an agreement with the assumptions resulted in Eqs. (38) and (39). The minimum excess number of molecules in the critical cluster $\overline{\Delta n_{*}}$ (attained at the crossover) increases with temperature. The parameter $\kappa$ also increases with $T$; the value $\kappa=1$, at which the qualitative change in the behavior of the barrier scaling function occurs, corresponds for argon to $T / T_{c} \approx 0.39$. A general conclusion is that the temperature rise shifts the boundary between the CNT domain and the spinodal domain in the direction of the former.

In experiment one measures directly the nucleation rate $J$ as a function of temperature and supersaturation (see Ref. 28 ), hence it is convenient to relate $\Gamma$ to $J$. Assuming following $^{12}$ that the kinetic prefactor $K$ is well approximated by the CNT we find from Eq. (4)

$$
\frac{J(T ; \eta)}{J^{\mathrm{cl}}(T ; \eta)}=\exp \left\{\Lambda\left[\frac{1-\Gamma(\eta ; T)}{\eta^{2}}\right]\right\} \text {. }
$$

It is instructive to study this ratio at a fixed value of $J^{\mathrm{cl}}$. With this in mind we choose the quench depth $\eta=\eta_{1}$ corresponding to a typical value of $J^{\mathrm{cl}}\left(\eta_{1}\right)=1 \mathrm{~cm}^{-3} \mathrm{~s}^{-1}$. This implies using Eqs. (4) and (5) that $\eta_{1}$ satisfies,

$$
\frac{\Lambda}{\eta_{1}^{2}}=\Psi+2 \eta_{1} \ln S_{\mathrm{sp}}
$$

where

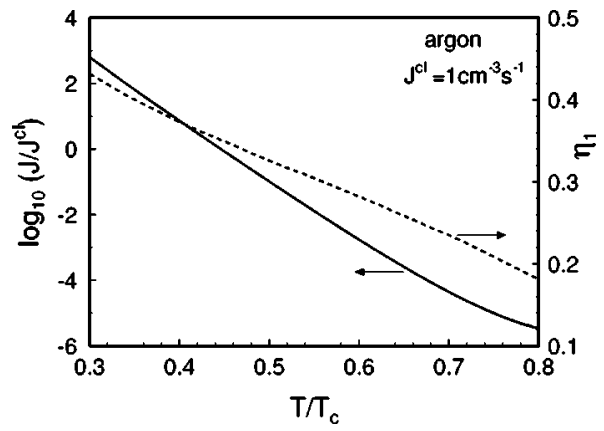

FIG. 5. $\log _{10}\left(J / J^{\mathrm{cl}}\right)$ for argon as given by Eq. (48) (solid line); the quench depth $\eta_{1}$ corresponding to $J^{\mathrm{cl}}=1 \mathrm{~cm}^{-3} \mathrm{~s}^{-1}$ [Eq. (49)] is shown on the right $y$ axis (dashed line).

$$
\Psi(T)=\ln \left[\frac{p_{\text {sat }}^{2}}{\rho_{\mathrm{eq}}^{l}\left(k_{\mathrm{B}} T\right)^{2}} \sqrt{\frac{2 \gamma_{\infty}}{\pi m_{1}}}\right] .
$$

Figure 5 shows the ratio $J\left(T ; \eta_{1}\right) / J^{\mathrm{cl}}\left(T ; \eta_{1}\right)$ for the same temperature interval as in Fig. 4 (solid line); the value of $\eta_{1}$ is shown on the right $y$ axis (dashed line).

Thus, for argon the CNT underestimates the nucleation rate at lower temperatures for all supersaturations. At higher temperatures the CNT overestimates $J$ for (relatively) small $S$ and underestimates $J$ for (relatively) high $S$. This result is an agreement with the density functional calculations of nucleation in Lennard-Jones fluids. ${ }^{26}$ It is not clear whether this tendency remains true for more complex substances. This is a problem to be studied in future work.

At this stage comparison with experiment can be only qualitative since for the spinodal properties we used the simplest van der Waals equation of state. It would be desirable to verify the predictions of the theory for the substances for which the thermodynamic spinodal can be accurately estimated.

\section{ACKNOWLEDGMENTS}

It is a pleasure to thank M. E. H. van Dongen, V. Holten, and D. Labetskii for helpful discussions. The author is indebted to H. Bruining, L. Dyadkin, E. Slob, and D. Smeulders for valuable comments.

\section{APPENDIX: SUPERSATURATED VAPOR DENSITY AND THERMODYNAMIC SPINODAL FOR VAN DER WAALS FLUIDS}

In reduced units $\rho^{*}=\rho / \rho_{c}, T^{*}=T / T_{c}, p^{*}=p / p_{c}$ the van der Waals equation of state reads ${ }^{13}$

$$
p^{*}=-3 \rho^{* 2}+\frac{8 \rho^{*} T^{*}}{3-\rho^{*}} .
$$

Solving this cubic equation for the vapor density $\rho^{* v}\left(p^{*}\right)$ we obtain using the standard methods: ${ }^{19}$

$$
\rho^{* v}\left(p^{*}, T^{*}\right)=1+2 \sqrt{1-\left(\frac{8 T^{*}+p^{*}}{9}\right)} \cos \left(\alpha+\frac{2 \pi}{3}\right),
$$


where $\alpha$ is given by

$$
\cos (3 \alpha)=\frac{1-\left(\frac{4 T^{*}-p^{*}}{3}\right)}{\left[1-\left(\frac{8 T^{*}+p^{*}}{9}\right)\right]}
$$

The spinodal equation $\partial p^{*} / \partial \rho^{*}=0$ is

$$
T^{*}=\frac{\rho^{*}}{4}\left(3-\rho^{*}\right)^{2} .
$$

Solving for the spinodal vapor density $\rho_{\mathrm{sp}}^{*^{v}}$ we obtain

$$
\rho_{\mathrm{sp}}^{*^{v}}\left(T^{*}\right)=2-2 \cos \left(\frac{1}{3} \beta\right), \quad \beta=\arccos \left(1-2 T^{*}\right) .
$$

The parameter $\omega$ defined in Eq. (42) is

$$
\omega=6 \rho_{\mathrm{sp}}^{*^{v}}-\frac{48 T^{*} \rho_{\mathrm{sp}}^{*^{v}}}{\left(3-\rho_{\mathrm{sp}}^{*^{v}}\right)^{3}} .
$$

Substitution of Eq. (A2) into Eq. (A1) yields the vapor pressure at the spinodal,

$$
p_{\mathrm{sp}}^{*^{v}}=\frac{8\left[4 T^{*}-3 \cos \left(\frac{1}{3} \beta\right)+3 \cos \left(\frac{2}{3} \beta\right)\right] \sin ^{2}\left(\frac{1}{6} \beta\right)}{1+2 \cos \left(\frac{1}{3} \beta\right)},
$$

from which the maximum supersaturation is

$$
\begin{aligned}
S_{\mathrm{sp}}\left(T^{*}\right)= & \frac{p_{\mathrm{sp}}^{*^{v}}\left(T^{*}\right)}{p_{\mathrm{sat}}^{*}\left(T^{*}\right)} \\
= & {\left[\frac{1}{p_{\mathrm{sat}}^{*}\left(T^{*}\right)}\right] } \\
& \times\left\{\frac{8\left[4 T^{*}-3 \cos \left(\frac{1}{3} \beta\right)+3 \cos \left(\frac{2}{3} \beta\right)\right] \sin ^{2}\left(\frac{1}{6} \beta\right)}{1+2 \cos \left(\frac{1}{3} \beta\right)}\right\} .
\end{aligned}
$$

${ }^{1}$ R. McGraw and A. Laaksonen, J. Chem. Phys. 106, 5284 (1997); V. Talanquer, ibid. 106, 9957 (1997); K. Koga and X. C. Zeng, ibid. 110, 3466 (1999); I. Kusaka, ibid. 118, 5510 (2003); 119, 1808 (2003).

${ }^{2}$ D. Kashchiev, J. Chem. Phys. 118, 1837 (2003).

${ }^{3}$ J. W. Schmelzer and V. G. Baidakov, J. Chem. Phys. 119, 10759 (2003).

${ }^{4}$ H. Gould and W. Klein, Physica D 66, 61 (1993).

${ }^{5}$ J. W. Cahn and J. E. Hilliard, J. Chem. Phys. 28, 258 (1958); 31, 688 (1959).

${ }^{6}$ J. S. Langer, Ann. Phys. (N.Y.) 41, 108 (1967); 54, 258 (1969).

${ }^{7}$ W. Klein, Phys. Rev. Lett. 47, 1569 (1981).

${ }^{8}$ C. Unger and W. Klein, Phys. Rev. B 29, 2698 (1984).

${ }^{9}$ A. Z. Patashinskii and B. I. Shumilo, Sov. Phys. JETP 50, 712 (1979); Sov. Phys. Solid State 22, 655 (1980).

${ }^{10}$ J. W. Gibbs, The Scientific Papers of J. W. Gibbs (Ox Bow, Woodbridge, NJ, 1993).

${ }^{11} \mathrm{~J}$. S. Rowlinson and B. Widom, Molecular Theory of Capillarity (Clarendon, Oxford, 1982).

${ }^{12}$ D. W. Oxtoby, J. Phys.: Condens. Matter 4, 7627 (1992).

${ }^{13}$ V. I. Kalikmanov, Statistical Physics of Fluids. Basic Concepts and Applications (Springer, Berlin, 2001).

${ }^{14}$ D. W. Oxtoby and D. Kashchiev, J. Chem. Phys. 100, 7665 (1994); D. Kashchiev, ibid. 76, 5098 (1982).

${ }^{15}$ Recently Bowles et al. (Ref. 16) proved the generalized version of the nucleation theorem which extends its applicability to all equilibrium systems containing local density inhomogeneities.

${ }^{16}$ R. K. Bowles, D. Reguera, Y. Djikaev, and H. Reiss, J. Chem. Phys. 115, 1853 (2001)

${ }^{17}$ D. W. Oxtoby and R. Evans, J. Chem. Phys. 89, 7521 (1988).

${ }^{18}$ L. D. Landau and E. M. Lifshitz, Statistical Physics (Pergamon, Oxford, 1969).

${ }^{19}$ G. A Korn and T. M. Korn, Mathematical Handbook (McGraw-Hill, New York, 1968).

${ }^{20}$ Yu. B. Rumer and M. S. Rivkin, Thermodynamics, Statistical Physics and Kinetics (Moscow, Nauka, 1977) (in Russian).

${ }^{21}$ A. J. Bray, Adv. Phys. 43, 357 (1994).

${ }^{22}$ J. J. Binney, N. J. Dowrick, A. J. Fisher, and M. E. J. Newman, The Theory of Critical Phenomena (Clarendon, Oxford, 1995).

${ }^{23}$ M. Struwe, Variational Methods: Application to Nonlinear Partial Differential Equations and Hamiltonian Systems (Springer, Berlin, 2000).

${ }^{24}$ P. M. Chaikin and T. C. Lubensky, Principles of Condensed Matter Physics (Cambridge University Press, Cambridge, 1995).

${ }^{25}$ S. B. Kiselev, Physica A 269, 252 (1999).

${ }^{26}$ X. C. Zeng and D. W. Oxtoby, J. Chem. Phys. 94, 4472 (1991).

${ }^{27}$ R. C. Reid, J. M. Prausnitz, and B. E. Poling, The Properties of Gases and Liquids, 4th ed., (McGraw-Hill, New York, 1987).

${ }^{28}$ C. C. M. Luijten, P. Peeters, and M. E. H. van Dongen, J. Chem. Phys. 111, 8535 (1999). 